\title{
GMAW SHIELDING GAS FLOW OPTIMISATION BY REFINEMENT OF NOZZLE GEOMETRY
}

\author{
S.W. Campbell ${ }^{1}$, A.M. Galloway ${ }^{1}$, N.A. McPherson ${ }^{2}$ \\ ${ }^{1}$ Dept. of Mechanical \& Aerospace Engineering, University of Strathclyde, Glasgow, UK \\ ${ }^{2}$ BAE Systems Surface Ships Limited, Glasgow, UK \\ GMAW, CFD, Shielding gas, Cross draft, Flow profile
}

\begin{abstract}
With an ongoing demand to improve the efficiency of the gas metal arc welding process, steps are being taken to reduce the shielding gas consumption. However, sufficient shielding gas coverage of the weld region is essential for the generation of high quality welds, and drafts can be detrimental to its efficiency. In industry, the general practise to ensure coverage is to increase the shielding gas flow rate, however, too high a flow rate can induce undesirable turbulence in the shielding gas column, whilst adding unnecessary cost to the process. A simplified computational fluid dynamics model has been generated, and validated through extensive experimental trials, to accurately model the shielding gas flow when subjected to the adverse effects of cross drafts. Several nozzle geometry changes have been investigated with the aim of improving the shielding gas column's resistance to drafts, eliminating the requirement to increase the shielding gas flow rate.
\end{abstract}

\section{Introduction}

The shielding gas is a fundamental component of any gas shielded welding process, with effective shielding a prerequisite for good quality welds. There are a number of shielding gases commonly used, their primary purpose to protect the metal transfer and molten weld metal from contamination by atmospheric gases. Each gas has its own specific properties, which give them the ability to influence the mode of metal transfer, cleaning action, penetration level and solidified weld geometry. There are a number of factors affecting the shielding gas selection including the welding process, material to be welded and the economic availability of each gas. In the European shipbuilding industry there is a preference for an $80 \%$ argon / $20 \%$ carbon dioxide mixture for structural steel.

The correct shielding gas flow rate is essential for providing adequate protection to the weld metal. Welding operatives generally have a mindset that more shielding gas will result in better protection for the welding region and surveys have shown that the shielding gas flow rate be set as high as $36 \mathrm{l} / \mathrm{min}$ in shipyards, with an average flow rate of $24-26 \mathrm{l} / \mathrm{min}$. However, too low a flow rate could allow atmospheric gases to contaminate the weld region, whilst too high a flow rate can lead to turbulence in the shielding gas column [1], thus encouraging the intermixing of atmospheric gases.

Several studies have investigated the effects of reducing the shielding gas flow rate. Campbell et al. [2,3] investigated the effects of using gas saving devices in order to reduce the shielding gas consumption, and determined that the flow rate could be reduced to $61 / \mathrm{min}$ without detriment to weld quality in a draft free environment.

Drafts however, present a significant threat to the shielding gas efficiency and studies [3-7] have been conducted to determine safe working environmental conditions. Beyer et al. [4] used shadowgraphy in order to experimentally visualise the shielding gas flow for a variety of 
shielding gas flow rates and cross draft velocities, and resulted in the shielding gas flow rate in production welding being reduced to $12 \mathrm{l} / \mathrm{min}$. Campbell et al. [5] also generated an artificial neural network to predict the final weld quality for a range of weld inputs including nozzle outlet diameter, alternating gas frequency and flow rate of each shielding gas.

This study focuses on the development of a computational fluid dynamics (CFD) model to simulate the shielding gas flow for both a conventional nozzle and those incorporating a variety of geometry changes, including reducing the outlet diameter [6] and the use of restricting end and internal plates [7]. In doing so, determining the susceptibility of each configuration to a range of cross draft velocities.

\section{CFD Model Generation}

A 3D, multi-physics, transient state model was developed to simulate various nozzle geometry changes using the CFD solver Fluent, and takes into account buoyancy forces, convection and radiation. The model geometry was constructed in Gambit to replicate the experimental setup. The nozzle was centrally positioned, $10 \mathrm{~mm}$ above a $250 \times 500 \times 8 \mathrm{~mm}$ plate (width $\mathrm{x}$ length $\mathrm{x}$ depth), in a volume of fluid $600 \times 1000 \times 200 \mathrm{~mm}$, encapsulating the nozzle and plate in order to model all flow development of interest. A simplification of the welding arc was assumed which involved a $12 \mathrm{~mm}$ diameter hemisphere being positioned adjacent to the plate surface, directly beneath the welding nozzle, as shown in Figure 1. The density of the mesh was increased in the area between the nozzle outlet and the plate surface, as this was the main area of interest.

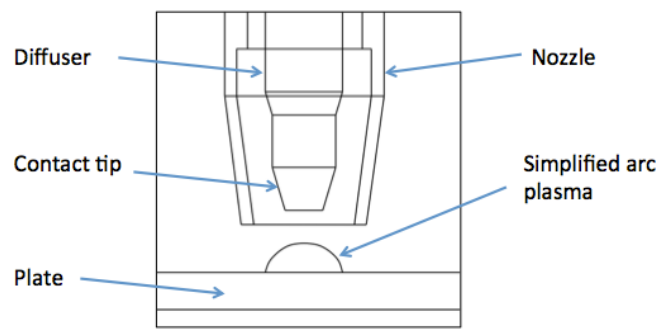

Figure 1: CFD nozzle geometry showing simplified arc plasma

The simplification of the arc plasma was based upon several studies conducted in the gas tungsten arc welding process which showed that the shielding gas composition, welding current, arc length and electrode geometry were the dominant factors influencing the arc pressure distribution [8-10]; all of which are constants in the present study. Furthermore, a study [11] showed that the shielding gas pressure has negligible effect on the arc pressure distribution. This led to the assumption that the arc pressure distribution in this study can be assumed constant from which various forces (e.g. arc force, arc shear stresses and Lorentz magnetic forces) can be numerically derived $[12,13]$. While this simplification of the arc plasma may be inadequate for the accurate modelling of certain weld aspects, for the case of shielding gas flow simulation it has been shown to be sufficiently accurate through validation with shadowgraphy and radiographic examination of the solidified weld $[4,6,7]$.

The cross draft of air was modelled as a $25 \mathrm{x} 40 \mathrm{~mm}$ (height $\mathrm{x}$ width) velocity inlet on the surface of the 3D domain, perpendicular to the direction of travel. This generated a uniform, laminar airflow as this best correlated with the conditions produced during experimental 
validations, the velocity of which could be measured in the region between the nozzle and plate surface.

\section{Experimental Validation}

Experimental validation was performed through means of bead on plate welds, on $8 \mathrm{~mm}$ think DH36 steel. These welds were deposited using an automatic welding rig, which moved a $250 \mathrm{x}$ $500 \times 8 \mathrm{~mm}$ plate at a set velocity of $3.2 \mathrm{~mm} / \mathrm{s}$, corresponding with the plate velocity set in simulations. The plate is moved below a, centrally located, stationary GMAW nozzle that is situated $10 \mathrm{~mm}$ above the plate surface. The modified welding nozzles used in the experimental validation are shown in Figure 2, and had a coating of anti-spatter applied prior to welding to prevent a build up of spatter. $1.2 \mathrm{~mm}$ diameter SF-1A flux cored filler wire with a feed speed of $120 \mathrm{~mm} / \mathrm{s}$ was maintained constant throughout. As with the computational trials, the shielding gas used during the experimental validation was $80 \% \mathrm{Ar} / 20 \% \mathrm{CO}_{2}$, the gas flow rate of which was monitored using a dedicated welding monitor. The arc voltage and current was also measured throughout the trials using a pre-calibrated portable arc monitoring system. This returned average welding parameters of $24.6 \mathrm{~V}$ and $225 \mathrm{~A}$ for arc voltage and current respectively.
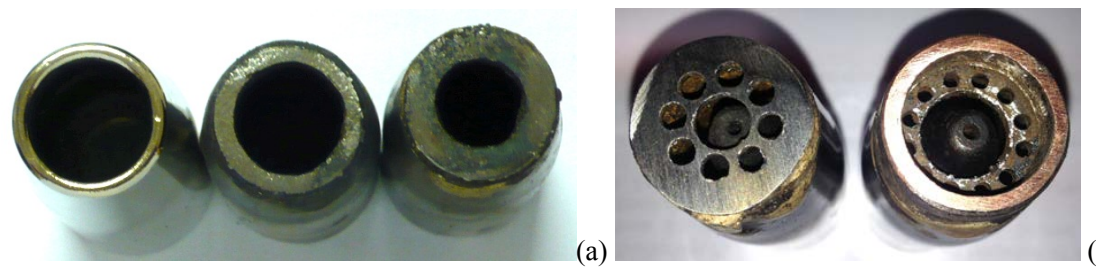

Figure 2: Weld nozzle geometries showing (a) conventional $16 \mathrm{~mm}$ nozzle, restricted $14 \mathrm{~mm}$ nozzle and restricted $11 \mathrm{~mm}$ nozzle, and (b) end plate restriction and internal plate restriction

A cross draft was produced by means of a compressed air supply passing through an adjustable laminar flow device, with a $20 \times 25 \mathrm{~mm}$ outlet. This was positioned $300 \mathrm{~mm}$ from the centre of the welding nozzle and directed towards the shielding gas column at an angle 90 degrees to the weld direction as shown in Figure 3. Using a pre-calibrated hot-wire anemometer, the draft velocity in the region between the welding nozzle and plate surface was measured, enabling it to be set to the appropriate velocity for each trial.

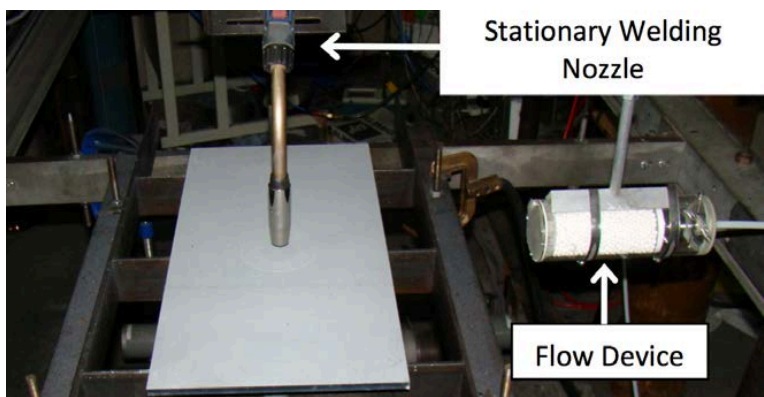

Figure 3: Experimental setup showing flow device to induce cross draft 


\section{Results and Discussion}

Each nozzle geometry change was subjected to a cross draft velocity of $0-8 \mathrm{mph}$, in $1 \mathrm{mph}$ increments, with a shielding gas flow rate of 5, 10, 15 and $18 \mathrm{l} / \mathrm{min}$.

A grading system for the flow profile was chosen based on an experimental weld width of 15 $\mathrm{mm}$ : profiles with $100 \%$ shield gas concentration on the plate surface to a distance of $\geq 8 \mathrm{~mm}$ on both sides of the nozzle centreline would produce a good quality weld. Table 1 shows the CFD classification using this grading system, whilst Figure 4 show examples of the shielding gas column when subjected to varying cross draft velocities.

The experimental validation weld quality was evaluated using radiographic inspection, and graded according to the amount of porosity present. Green indicates a clean weld free from porosity and a pass, yellow indicates a borderline result free from harmful porosity and red indicates a fail due to a highly porous weld. The radiographic inspections results are shown in Table 2.

The respective grading systems were found to produce an agreement in $90 \%$ of the cases with includes experimental errors, such as a fail for the $14 \mathrm{~mm}$ nozzle for a shielding gas flow rate of $5 \mathrm{l} / \mathrm{min}$ with no cross draft, and a pass for the $14 \mathrm{~mm}$ nozzle for a shielding gas flow rate of 10 $1 / \mathrm{min}$ and a $7 \mathrm{mph}$ cross draft, decreasing this percentage. Based on the surrounding results, these should both be the opposite of the radiographic examination and therefore in agreement with the CFD classification.

It can be observed that as the outlet diameter of the nozzle decreases, the shielding gas columns resistance to the adverse effects of the cross draft increases. This can be explained due to conservation of mass, as the shielding gas is flowing through a narrower orifice, the velocity has to increase in order to maintain mass flow. Spatter build-up on the weld nozzle is unavoidable in practice, although it can be minimised by good control of the welding parameters. The progressive restriction of the nozzle by spatter can therefore be beneficial against the effect of cross drafts to some extent, provided that the build-up is approximately uniform around the circumference of the nozzle and the overall flow is not excessively restricted.

The other geometry changes, i.e. the end and internal plates, did not show the same improvements to cross draft resistance as simply narrowing the nozzle exit diameter to $11 \mathrm{~mm}$. The end plate forces the shielding gas through a reduced cross-sectional area, thus increasing its velocity at the nozzle exit, and as a result improved on the performance of the conventional 16 $\mathrm{mm}$ nozzle. Although the internal plate increased the shielding gas velocity through the restriction, upon reaching the nozzle exit the shielding gas had lost most of the momentum gained. The $6 \mathrm{~mm}$ from the inserted plate to the nozzle exit was enough to allow the shielding gas to expand out into the larger cross sectional area of the nozzle, reducing its exit velocity.

The experimental measurements and CFD analysis have developed conditions in which the shielding gas flow rate can be effectively reduced, and without detriment to weld quality. In a typical shipyard, BAE Systems Surface Ships Ltd. were using an average shielding gas flow rate of 24-26 1/min against a process setting of $18 \mathrm{l} / \mathrm{min}$ with a $16 \mathrm{~mm}$ nozzle. Studies [2-7] have provided confidence that the shielding gas flow rate can be reduced with no loss of coverage and has allowed for flow controllers set at $12 \mathrm{l} / \mathrm{min}$ to be installed in the BAE Systems Govan shipyard. To date no defects have been found, and the predicted $50 \%$ shielding gas reduction will yield savings in the region of $£ 300 \mathrm{k}$ per annum. There will also be a further saving with a migration to an $11 \mathrm{~mm}$ nozzle, although spatter build-up may be a limiting factor. 


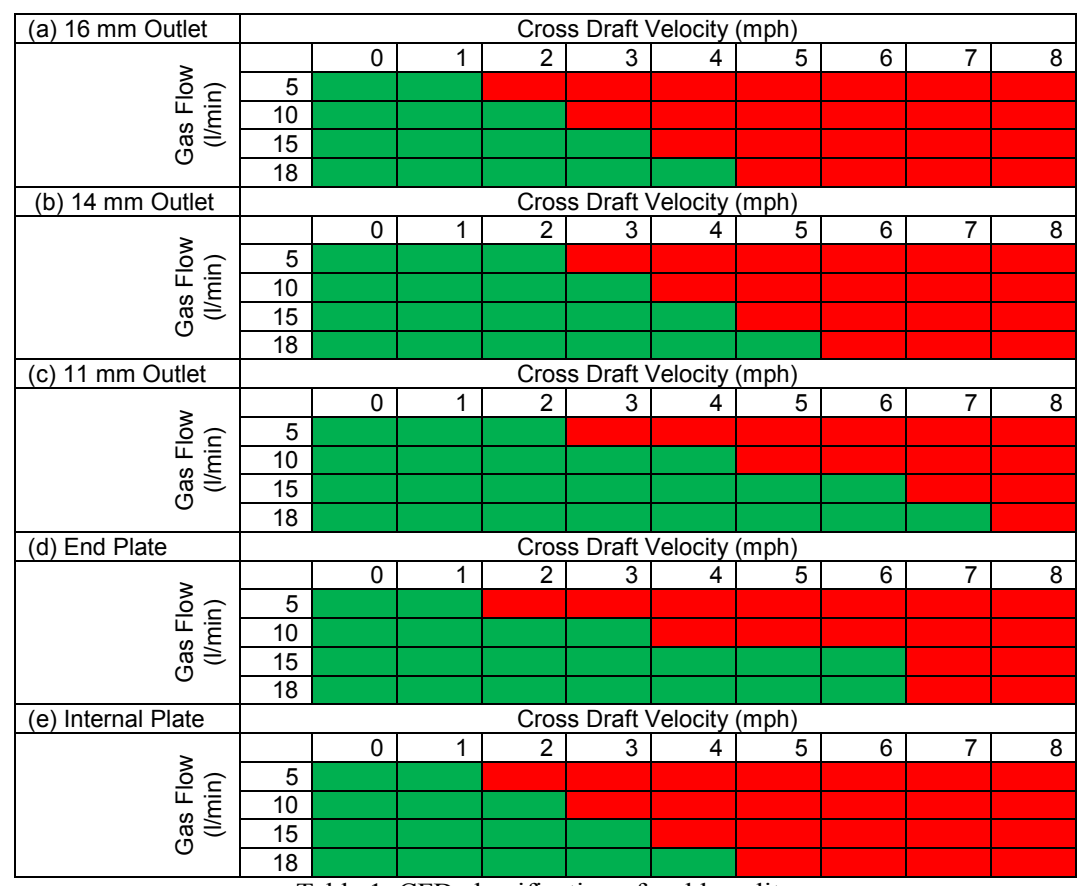

Table 1: CFD classification of weld quality
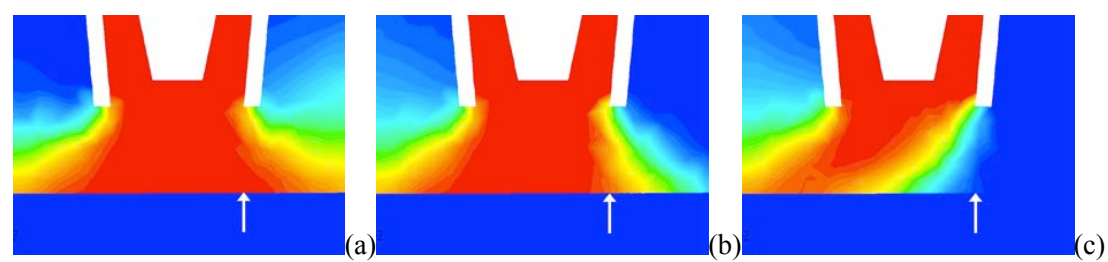

Figure 4: Selection of $16 \mathrm{~mm}$ nozzle CFD predictions at $10 \mathrm{l} / \mathrm{min}$ and (a) $1 \mathrm{mph}$, (b) $3 \mathrm{mph}$ and (c) 5 mph cross drafts 


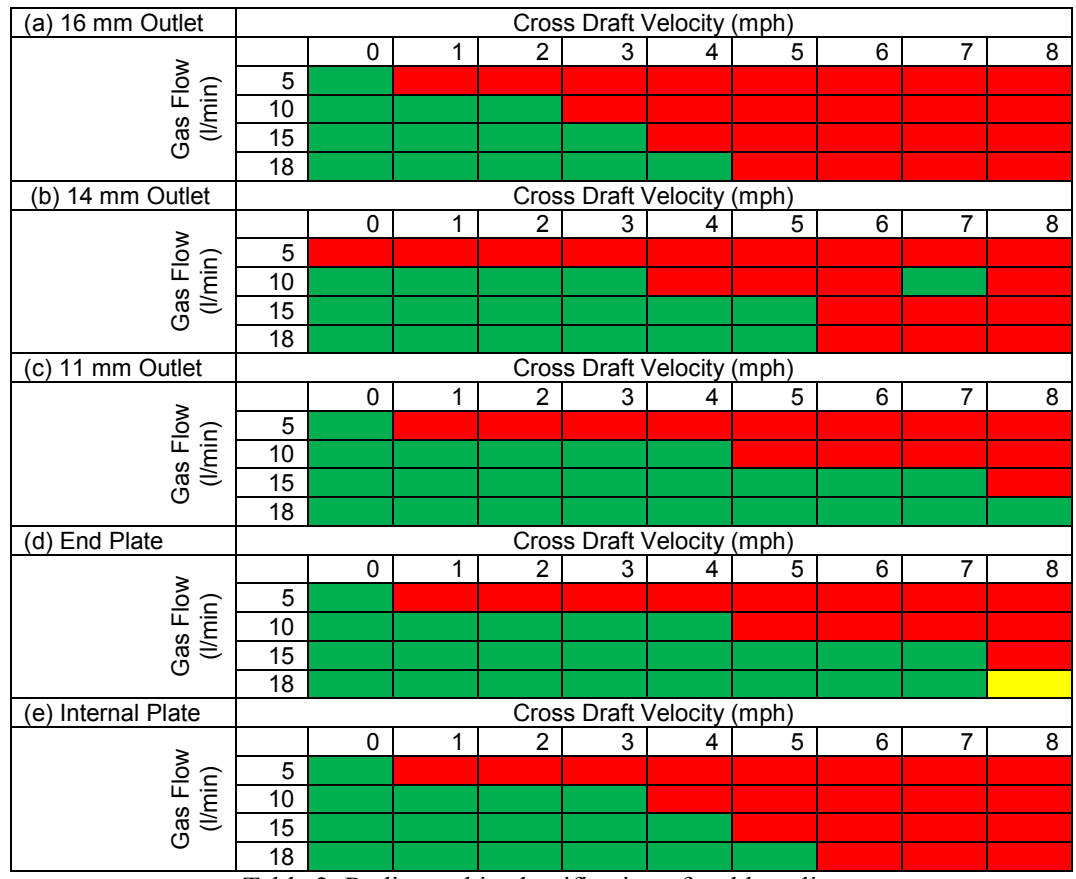

Table 2: Radiographic classification of weld quality

\section{Conclusions}

A simplified CFD model has been developed to determine the effects of geometry changes in the welding nozzle on the shielding gas flow coverage during GMAW. The conclusions may be summarised as follows:

- Both the CFD simulations and experimental validation trials have highlighted the adverse affect of cross drafts, and have indicted safe working conditions for reducing the shielding gas flow rate.

- A comparison of CFD simulations and experimental validation trials has indicated that the arc plasma simplification is sufficient in predicting the shielding gas flow profile.

- It was determined that the $11 \mathrm{~mm}$ restriction performed best due to the high increases in shielding gas velocity achieved.

- The other geometry changes, i.e. the end and internal plates, did not show the same level of cross draft resistance as simply narrowing the nozzle exit diameter to $11 \mathrm{~mm}$.

- A 50\% shielding gas reduction has been implemented in the BAE Systems Govan shipyard, which will yield savings in the region of $£ 300 \mathrm{k}$ per annum. 


\section{Acknowledgements}

The authors would like to acknowledge the funding provided by BAE Systems Surface Ships Limited, which has made this research possible.

In addition, the authors would like to acknowledge the conference funding provided by the Scottish Association for Metals and Regula Systems AB.

\section{References}

1. K. Lyttle and G. Stapon: 'Simplifying shielding gas selection', Practical Welding Today, 2005, 9(1).

2. S.W. Campbell, A.M. Galloway and N.A. McPherson: ' Techno-economic evaluation of reducing shielding gas consumption in GMAW whilst maintaining weld quality', International Journal of Advanced Manufacturing Technology, 2012, 63, 975-985.

3. S.W. Campbell, A.M. Galloway and N.A. McPherson: ' A potential solution to GMAW flow optimisation', Proceedings of the $9^{\text {th }}$ International Conference on Trends in Welding Research, 4-8 June 2012, Chicago, USA. ASM International, 453-460.

4. V. Beyer, S.W. Campbell, G.M. Ramsey, A.M. Galloway, A.J. Moore and N.A. McPherson: 'Systematic study of the effect of cross-drafts and nozzle diameter on shield gas coverage in MIG welding', Science and Technology of Welding and Joining, Under review

5. S.W. Campbell, F.H. Ley, A.M. Galloway and N.A. McPherson: 'ANN optimisation of shielding gas flow rate in GMAW subjecting to cross drafts when using alternating shielding gases', Journal of Manufacturing Science and Engineering, Under review.

6. G.M. Ramsey, A.M. Galloway, S.W. Campbell, N.A. McPherson and T.J. Scanlon: 'A computational fluid dynamics analysis of the effect of side draughts and nozzle diameter on shielding gas coverage during gas metal arc welding', Journal of Materials Processing Technology, 2012, 212(2012), 1694-1699.

7. G.M. Ramsey, S.W. Campbell, A.M. Galloway and N.A. McPherson: 'A computational fluid dynamic analysis of the effect of weld nozzle geometry changes on shielding gas coverage during gas metal arc welding, Journal of Manufacturing Science and Engineering, under review.

8. M.L. Lin and T.W. Eagar: 'Pressures produced by gas tungsten arcs', Metallurgical Transactions B, 1986, 17B, 601-607.

9. S.W. Campbell, A.M. Galloway and N.A. McPherson: 'Arc pressure and weld metal fluid flow whilst using alternating shielding gases - part 1: arc pressure measurement', Science and Technology of Welding and Joining, Under review.

10. H.G. Fan and Y.W. Shi: 'Numerical simulation of the arc pressure in gas tungsten arc welding', Journal of Materials Processing Technology, 1996, 61(1996), 302-308.

11. H.-S. Ham, D.-S. Oh and S.-M. Cho: 'Measurement of arc pressure and shield gas pressure effect on surface of molten pool in TIG welding', Science and Technology of Welding and Joining, 2012, 17(7), 594-600.

12. D.-S. Oh, Y.-S. Kim and S.-M. Cho: 'Derivation of current density distribution by arc pressure measurement in GTA welding', Science and Technology of Welding and Joining, 2005, 10(4), 442-446.

13. S.W. Campbell, A.M. Galloway and N.A. McPherson: 'Arc pressure and weld metal fluid flow whilst using alternating shielding gases - part 2: arc force determination', Science and Technology of Welding and Joining, Under review. 